\title{
Sex ratio variation in a eusocial mammal, the Damaraland mole-rat, Fukomys damarensis
}

\author{
Heike Lutermann ${ }^{1}$, Elissa Z. Cameron ${ }^{1,2}$, Nichola J. Raihani ${ }^{3}$ and Nigel C. Bennett ${ }^{1}$ \\ ${ }^{1}$ Mammal Research Institute, Department of Zoology \& Entomology, University of Pretoria, \\ Pretoria, 0002. South Africa \\ ${ }^{2}$ School of Zoology, University of Tasmania, Hobart, Australia. \\ ${ }^{3}$ Genetics, Evolution and Environment, University College London, WC1E 6BT
}

\begin{abstract}
Sex allocation theory predicts that mothers benefit from adjusting the sex ratio of their offspring in relation to their offspring's future reproductive success. In cooperative breeders, parents are expected to bias the sex ratio in relation to their current need for help and the benefit received from helpers of each sex as proposed by the local resource enhancement (LRE) and helper repayment hypotheses (HR). Consequently, as group size increases, sex ratios are expected to be biased towards the sex that is more likely to disperse to avoid competition as proposed in the local resource competition hypothesis (LRC). The current study aimed to investigate helper effects on breeder fecundity and offspring sex ratio adjustments in a eusocial mammal the Damaraland mole-rat (Fukomys damarensis). Both sexes equally contribute to helping in this species but breeding dispersal is male-biased. We found no evidence for helper effects on maternal body mass and litter size. Offspring sex ratio was not affected by maternal mass or litter size. However, the probability of male pups to be born increased with the number of female helpers and decreased with the number of male helpers. In addition, the number of male pups in a litter increased significantly with the number of female but not male helpers while no helper effects were apparent for the number of female pups born. Hence, our data suggest that the mechanisms underlying the LRE, HR and LRC may operate simultaneously in the study species.
\end{abstract}

Keywords: sex allocation; maternal investment; eusociality, Bathyergidae, cooperative breeding

\section{INTRODUCTION}

Fisher (1930) suggested that natural selection will favour equal investment of parents into offspring of both sexes. This is considered the cause of the balanced sex ratio observed in many animal populations. Sex allocation theory predicts that parents should adjust the sex of their offspring in relation to their ability to invest in that offspring and the resulting profitability of each sex to the parent (Trivers \& Willard, 1973). Such variation in investment is likely to be linked to maternal condition and in species where one sex is more costly to produce than the other or whose reproductive success is more condition-dependent, mothers in good condition should show a greater investment in this sex. In many species with a sexual dimorphism this is true for male offspring and in several of these species, females are more likely to have male offspring when in good condition while those in poorer condition tend to produce more female offspring (reviewed in Clutton-Brock \& Iason 1986; Cameron 2004).

Where one sex disperses while the other is philopatric, the profitability of each sex can be modified by local resource competition (LRC, Clark, 1978; Silk, 1984) or local resource enhancement (LRE, Gowaty \& Lennartz, 1985; Emlen, Emlen \& Levin, 1986). In cooperative breeders, where breeding individuals are assisted by non-reproductive helpers in the production of young, helping behaviour is often biased towards one sex, and members of the helping sex frequently delay dispersal (e.g. Koenig et al., 1992; Allainé, 2000). Conversely, successful dispersal and new group formation is usually biased towards the sex that helps less. The LRE hypothesis proposes that when parents gain more 
from helpers of one sex, they are expected to bias the sex ratio in relation to their current need for help (Pen \& Weissing, 2000), mediated by the benefit received from helpers of each sex and the prospect for successful dispersal by each sex. Alternatively, but not mutually exclusive the helper repayment (HR) hypothesis posits that females preferably produce the offspring sex that compensates them for their reproductive investment by helping (Gowaty \& Lennartz, 1985; Emlen et al., 1986; Lessells \& Avery, 1987). In other words, in newly formed groups with few helpers, sex ratios should be biased in favour of the helping sex. Once there are sufficient helpers, sex ratios should be biased towards dispersers to avoid LRC.

Evidence from cooperatively-breeding birds seems to support sex ratio adjustments as suggested by the LRE, HR and LRC hypotheses (e.g. Komdeur et al., 1997; Canestrari et al., 2012), with the extent of bias reflecting differences in benefits derived from male and female helpers (Griffin, Sheldon \& West, 2005). There have been fewer studies on mammals, but these tend to support the same trend, with more of the helping sex produced when helpers are absent (Allaine 2004; McNutt \& Silk 2008; Silk \& Brown 2008; Rapaport et al. 2013; but see MacLeod \& Clutton-Brock 2013). Where males disperse and success is dependent on the size of the dispersing cohort, mothers may bias larger litters towards sons (e.g. in lions, Packer \& Pusey, 1987). Therefore, initial evidence suggests that mammals may also show variation in support of the model's predictions.

Sherman et al., (1995) define eusociality, the most extreme form of cooperative breeding, by the presence of distinct physical castes where non-breeders are physiologically regulated. To date there has been no study on litter sex ratio variation in a eusocial mammal. The only two known species of eusocial mammals are both African mole-rats, subterranean hystricomorph rodents that are strongly constrained in their dispersal by the high energy demand for digging (Bennett \& Faulkes, 2000). In Damaraland molerats (Fukomys damarensis), natal philopatry typically results in colonies consisting of a breeding female and up to two genetically unrelated males and several litters of their offspring (Jarvis \& Bennett, 1993; Burland et al.,
2004) until favourable environmental characteristics make dispersal events possible. Observational and genetic evidence suggests that dispersal in Damaraland mole-rats is male-biased and closely related males may disperse together to found new colonies (Hazell et al., 2000; Burland et al., 2004). This strategy is likely to increase the success of dispersal as only $10 \%$ of all dispersal attempts are successful (Jarvis \& Bennett, 1993). Consequently, offspring unrelated to the breeding male as well as unrelated males can occur frequently in wild colonies while this is rarely true for females (Burland et al., 2004). At the same time, female helpers readily engage in matings when encountering unrelated males, such as when dispersing males enter a colony, despite being physiologically suppressed in the confines of their natal colony (Bennett, Faulkes \& Molteno, 1996). This can cause dramatically escalated aggression between breeding female and female helpers under laboratory conditions and field data suggest that this conflict is likely to result in dispersal of female helpers (Jarvis \& Bennett, 1993; Cooney \& Bennett, 2000).

Damaraland mole-rats have two physiologically distinct helper castes: frequent and infrequent workers. While both sexes are equally represented in both castes, the frequent helpers are smaller, sexually monomorphic and together perform more than $95 \%$ of the total work done by the colony, including finding food, maintaining the burrow system and nest building (Bennett \& Jarvis, 1988; Scantlebury et al., 2006). All individuals born in a group will be members of this caste once independent. Conversely, infrequent helpers perform less than $5 \%$ of the total work. They cease working and accumulate fat reserves, presumably for dispersal, and male infrequent helpers are significantly larger than females (Bennett \& Jarvis, 1988; Hazell et al., 2000; Scantlebury et al., 2006). Only some frequent workers become infrequent workers later in their life and it is unknown what predisposes an individual for this transition. An increasing workforce makes foraging more efficient and allows breeders to reduce their contributions to costly tasks such as digging (Bennett \& Jarvis, 1988; Sichilima et al., 2008). In addition, breeder life-expectancy is greatly improved for breeders in the genus 
Fukomys both in captivity and in the field (Dammann \& Burda, 2006; Dammann et al., 2011; Schmidt, Jarvis \& Bennett, 2013). This is possibly because the presence of helpers allows a greater investment in immune defence (Lutermann \& Bennett, 2008). In light of the male bias in dispersal patterns, producing female biased litters when group size is small may be beneficial for Damaraland breeders. This is because female helpers are more likely to be present for extended periods than the less philopatric male offspring. In other cooperatively breeding mammals, helpers have been shown to positively impact breeding female fitness, either through direct effects on litter size and offspring survival or through indirect effects on breeding female condition (Russell et al., 2003; Allainé, 2004; Rapaport et al., 2013). Despite the apparent positive effects of helpers on breeders' energy budgets and life expectancy, the fitness implications of helper number (both total as well as of a particular sex) for breeders in Damaraland mole-rats remain largely unexplored.

Here we investigate the possible benefits that breeding females derive from the presence of helpers as well as offspring sex ratio variation in captive colonies of eusocial Damaraland mole-rats. We firstly aimed to assess possible benefits of helping with regards to maternal condition (i.e. body mass) and the litter size at birth for the first time in the study species and predicted that female body mass and/or litter size increases with helper number. If such a benefit was predominately attributable to one helper sex, the number of helpers of this sex rather than group size would be expected to affect maternal condition. Secondly, we aimed to evaluate indirect (i.e. maternal mass and litter size) as well as direct effects of helpers (both of the total number as well as sex-specific) on offspring sex ratio. Since both sexes are helping and only frequent helpers are likely to be present in groups initially, we predicted in accordance with the LRE and HR hypotheses that in pairs and small groups, females would not produce sexbiased litters (Table 1). In contrast, both sexes may make the transition to the infrequent helper caste (significantly reducing their contribution to helping) but males in this caste are more likely to contribute to parent fitness by showing breeding dispersal. Consequently, we expected litters to be male-biased in large groups or those with a large number of female helpers in accordance with the LRC. Furthermore, we tested the hypothesis that females may produce a larger number of sons in larger litters as could be expected if the formation of male coalitions for breeding dispersal would be facilitated by the presence of same-age brothers.

\section{MATERIALS AND METHODS}

\section{Study Subjects}

The subterranean life-style of mole-rats makes it impossible to obtain detailed data required for this study from wild colonies. Consequently, we established captive colonies from adult $F$. damarensis captured between August 1984 and 1986 and between October 2008 and April 2009 using custom-made PVC Hickman live-traps (Hickman, 1979) in the field at Hotazel in the Northern Cape Province $\left(27^{\circ} 17^{\prime} \mathrm{S} 22^{\circ} 58^{\prime} \mathrm{E}\right)$ and Dordabis in Namibia $\left(22^{\circ} 58^{\prime} \mathrm{S} 17^{\circ} 41^{\prime} \mathrm{E}\right)$. A total of 44 and 35 individuals, respectively, were removed from the two sites. Captures were conducted by exposing tunnels in the vicinity of fresh mole-rat mounds and Hickman life-traps baited with sweet potato were placed at the entrances of those tunnels. Traps remained open for at least three days after the last individual had been captured to ensure that no further animals remained in a burrow system. Capture permits were obtained from Department of Nature Conservation Northern Cape and the Department of Environment and Tourism in Namibia. All captured individuals were housed with their colony mates (2-12 individuals) in plastic crates $(60 \mathrm{~cm} \times 30 \mathrm{~cm} \times 30 \mathrm{~cm})$ with wood shaving and paper towel as bedding and kept indoors during field trips but exposed to natural lighting. In the field and during transport to the laboratory the animals were maintained on a diet of geophytes (Dicadi sp.) and sweet potato provided ad libitum. Animals were transferred to artificial holding facilities in light- and temperature- $(12 \mathrm{~h}$ light/12h dark, $\left.25 \pm 1{ }^{\circ} \mathrm{C}\right)$ controlled rooms. A small plastic box served as a nest in which the animals slept. Wood shavings and paper towelling were provided as nesting material. The mole-rats were fed chopped sweet potato, pumpkin, carrots and apples daily. The mole-rats were initially paired as an unrelated male and 
female. Thereafter, the surviving offspring were left in the colony to simulate colony growth in wild populations. Twenty nine colonies were established and followed from 1984 to 1991 and from 2009 until June 2012 for between one and five generations (mean \pm SE: $2.38 \pm 0.27$ ) that survived until weaning at the age of four weeks. During 1984 to 1991 and October 2010 until June 2012 all individuals older than one month were weighed at regular intervals (weekly to monthly). In addition, we recorded the number of male and female helpers present in the colony when a litter was born. These procedures were approved by the Animal Ethics Committees of the Universities of Cape Town and Pretoria, respectively. Litter size was recorded for each litter born that survived until weaning and the sex of offspring was determined by visual investigation of the ano-genital distance and presence of penis which allows reliable sexing of pups shortly after birth (N.C. Bennett, personal observation). Although most pups were sexed before weaning we chose this time as cut-off point in order to avoid mortality induced by disturbance and handling of young pups. Offspring sex ratio (\% males) was calculated by dividing the number of male pups by litter size. Only litters for which all pups could be sexed were considered.

\section{Data Analyses}

We tested for skew in the overall pup sex ratio with a binomial test. Possible effects of helpers on maternal body mass were evaluated by employing generalized linear mixed models (GLMMs) to control for multiple litters from individual females $(\mathrm{n}=29)$. Body mass measurements were not always available for the time of conception but were available for all breeding females within two weeks of birth of a litter. Since maternal mass around conception ( \pm 90 days prior to birth) was highly correlated with post-natal mass for those breeding females for which both measurements were available $\left(\mathrm{R}_{\mathrm{S}}=0.833, \mathrm{n}=44, \mathrm{p}<0.0001\right)$, we used the body mass of breeding females measured within two weeks of the birth of a litter for analyses. As breeding female mass was not normally distributed and transformations were unsuccessful we fitted a GLMM with a Gammadistribution and a log-link function. Total group size or the numbers of male and female helpers, respectively were included as independent variables. This approach was chosen to test whether group size per se or only one helper sex influences fitness consequences and/or sex allocation. We explored the effects of maternal mass and helper number of each sex on litter size in a GLMM fitting a Poisson-distribution with a log-link function. Post-hoc pairwise comparisons were carried out for factors that significantly affected litter size using the least significant difference (LSD). We tested for the effects of breeding female mass, group size or helper number of each sex and litter size on offspring sex ratio with a GLMM with a binomial distribution and a logit-link function. In addition to sex ratio we tested for the effects of breeding female mass, group size or helper number of each sex and litter size on the number of male and female pups, respectively, born in a litter employing a GLMM with Poisson-distribution with a log-link function and the number of male and female pups, respectively, as dependent variables. For all GLMMs breeding female identity was fitted as random effect. We chose to employ both of these approaches as it allowed us to address the questions whether in addition to overall sex ratio adjustments females show evidence for an overproduction of male offspring in large litters. Data were analysed using SPSS (IBM Corp. SPSS statistics version 21, 2012) and results are reported as means \pm SE.

\section{RESULTS}

\section{Helper Effects on Breeder Fitness}

The body mass of breeding females varied widely and ranged from 68 to $185 \mathrm{~g}$ (130.2 \pm $3.3 \mathrm{~g}$ ). However, it did not vary significantly with either the number of male $\left(\mathrm{F}_{1,66}=0.024, \mathrm{p}=0.876\right)$ or female helpers $\left(\mathrm{F}_{1,66}=0.513, \mathrm{p}=0.476\right)$.

In total, 68 litters comprising 138 pups were recorded throughout the study for which litter size was known. Litter size varied from 1-5 pups $(2.12 \pm 0.12)$, with the majority containing more than one pup $(59.4 \%)$. It increased significantly with mass of the breeding female $\left(F_{1,65}=10.045, p=0.002\right.$, Figure 1) with females giving birth to litters of one pup being significantly lighter than those giving birth to larger litters (LSD: $\mathrm{p} \leq 0.009$ ). However, litter size did not vary significantly with either the 
number of male $\left(\mathrm{F}_{1,65}=0.233, \mathrm{p}=0.631\right)$ or female $\left(\mathrm{F}_{1,65}=0.006, \mathrm{p}=0.937\right)$ helpers in the group.

\section{Offspring Sex Ratios}

Overall, offspring sex ratios were female biased (60 male: 78 female; $44.3 \%$ male). However, this did not differ significantly from an expectation of parity $(\mathrm{p}=0.148)$. The offspring sex ratio was not significantly affected by a breeding female's body mass $\left(\mathrm{F}_{1,133}=2.746, \mathrm{p}=0.100\right)$ or litter size $\left(\mathrm{F}_{1,133}=1.696, \mathrm{p}=0.195\right)$. In contrast, it increased significantly with the number of female helpers $\left(F_{1,133}=10.143, p=0.002\right.$, Figure 2a $)$ while it decreased significantly with the number of male helpers $\left(\mathrm{F}_{1,133}=4.151, \mathrm{p}=0.044\right.$, Figure $\left.2 \mathrm{~b}\right)$. Similarly, offspring sex ratio increased significantly with group size $\left(\mathrm{F}_{1,134}=4.183\right.$, $\mathrm{p}=0.043$ ).

The body mass of the breeding female did not significantly affect the number of male pups in a litter $\left(\mathrm{F}_{1,63}=2.263, \mathrm{p}=0.137\right)$. Similarly, there was no significant effect of the number of male helpers on the number of male pups in a litter although this only just failed significance (estimate: $-0.129 \pm 0.065, \mathrm{~F}_{1,63}=3.936, \mathrm{p}=0.052$ ). However, the number of male pups increased significantly with the number of female helpers (estimate: $0.229 \pm 0.091, \mathrm{~F}_{1,63}=6.357, \mathrm{p}=0.014$ ) and litter size $\left(\mathrm{F}_{1,63}=16.175, \mathrm{p}<0.0001\right)$. In contrast, the number of female pups was neither affected by breeding female body mass $\left(\mathrm{F}_{1,63}=0.920, \mathrm{p}=0.341\right)$, the number of male $\left(\mathrm{F}_{1,63}=1.210, \mathrm{p}=0.275\right)$ or female helpers (estimate: $-0.211 \pm 0.108, \mathrm{~F}_{1,63}=3.797, \mathrm{p}=0.056$ ). However, the number of female pups increased significantly with litter size $\left(F_{1,63}=9.956\right.$, $\mathrm{p}=0.002)$. In contrast, neither the number of male $\left(\mathrm{F}_{1,64}=1.735, \mathrm{p}=0.193\right)$ nor female offspring $\left(\mathrm{F}_{1,64}=1.219, \mathrm{p}=0.274\right)$ was significantly affected by group size.

\section{DISCUSSION}

Our study did not find evidence for either indirect or direct effects of helpers on breeding females in Damaraland mole-rats. As in Alpine marmots (Marmota marmota) helper numbers did not increase breeding female mass (Allainé, 2004; Allainé \& Theuriau, 2004). In contrast, increases of breeding female mass with an increasing number of helpers have been reported for other cooperatively breeding mammals
(Bales et al., 2001; Russell et al., 2003). Direct helper effects on litter size on the other hand are rare and as in non-cooperative mammals, maternal mass was a key determinant of litter size (Russell et al., 2003; Allainé, 2004; Gilchrist, Otali \& Mwanguhya, 2004; Ryan et $a l ., 2012)$. Although non-breeding individuals exhibit a number of helping behaviours such as nest maintenance and foraging activity in captivity, we cannot entirely exclude the possibility that the reduced energetic demands for foraging as well as the ad libitum food availability in our study population may have masked possible helper effects on breeding females. However, helpers can affect maternal fitness in a number of ways and for several cooperatively breeding vertebrates the presence of helpers does significantly reduce inter-birth intervals which in turn can greatly increase the number of offspring produced (Solomon \& French, 1997; Russell et al., 2003; Russell, 2004). A similar mechanism could be acting in Damaraland mole-rats as well. In addition, helpers may substantially increase breeder survival by allowing a greater investment in immune defence (Dammann \& Burda, 2006; Lutermann \& Bennett, 2008; Dammann et al., 2011) and this could translate in a larger number of litters being produced throughout a breeding female's lifetime.

In accordance with reports from other mole-rat species we found no evidence for a biased offspring sex ratio at a population level (Begall \& Burda, 1998; Bennett \& Faulkes, 2000). Conversely, markedly female biased offspring sex ratios have been reported for Giant mole-rats (Fukomys mechowi) in captivity. The cause for this bias remains unclear and in the wild Giant mole-rats exhibit no sex bias among adults (Kawalika \& Burda, 2006). A significant male bias was observed in wild Damaraland mole-rats among large individuals (e.g. breeders and infrequent helpers) but not for smaller nonbreeders (Bennett \& Faulkes, 2000) supporting the hypothesis that sex ratios at birth are unlikely to be sex biased at a population level in the study species. The lack of sex bias in smaller nonbreeders (that are likely frequent workers) suggests that both sexes are equally costly to produce (Fisher, 1930). This is furthermore supported by the lack of sexual dimorphism in 
this caste and the comparable growth rates offspring of both sexes exhibit until weaning (Bennett et al., 1991; Bennett \& Navarro, 1997). Additional corroboration of the hypothesis that both sexes are equally costly to produce for a female is the lack of evidence for a condition dependent sex ratio adjustment in the study species as suggested by Trivers \& Willard, (1973). The slow growth of mole-rats means that adult weight is achieved long after weaning and breeding is affected by environmental rather than maternal effects (Bennett \& Faulkes, 2000), hence this result is not unexpected.

In contrast, the GLMM clearly indicated direct helper effects on litter sex ratios and the number of male pups born. Damaraland molerats are unusual among cooperatively breeding mammals in that both sexes are contributing equally to helping while they belong to the infrequent helper caste. Consequently, in smaller colonies (which are unlikely to contain infrequent helpers) there is little incentive to favour one offspring sex over the other. However, some frequent helpers of both sexes eventually make the transition into the infrequent helper caste were they bulk up weight probably by increasing their food intake (Bennett \& Jarvis, 1988; Scantlebury et al., 2006). The probability of male pups being born increased with the number of female helpers while it tended to decreased with the number of male helpers present. This finding partially supports the LRE and HR and LRC hypothesis (Table 1). Similarly mixed sex ratio allocation strategies have been reported for other mammal species (Allainé et al., 2000; Isaac, Krockenberger \& Johnson, 2005; Dušek, Bartoš \& Sedláček, 2011) have been reported for other species and indicate that this might be widespread among mammals.

Interestingly, it was the number of helpers of a certain sex rather than group size that affected offspring sex. This suggests that female breeders are able to detect the sexual composition of the workforce and adjust offspring sex ratio accordingly to balance benefits and costs. The probability of female offspring being born is greater in groups with few female helpers when resource competition should be low but the benefits of helpers should be high (Gowaty \& Lennartz, 1985; Emlen et al., 1986). With an increasing number of female helpers present the benefits accrued by philopatric helpers may be outweighed by the increasing LRC and the probability of male offspring being born increases and their higher dispersal rates would result in reduced competition (Clark, 1978; Silk, 1984). The mechanism behind such a sex ratio adjustment remains to be explored. However, it is possible that both food availability and/or increases in corticosteroid concentrations may contribute as has been suggested by others (Cameron, 2004; Ryan et al., 2012). Nevertheless, under the ad libitum conditions of the laboratory food constraints can be assumed to play a minor role. At the same time conflict-related increases in corticosteroid concentrations, as can be expected under the assumption of the LRC hypothesis, have been shown to result in male biased litters (Ryan et al., 2012). This is further supported by the observation that the number of pups of the dispersing sex (males) but not the philopatric sex was affected by the number of female helpers present in a group. At the same time, the observation that litter size affected both the number of male and female pups while it did not affect the sex ratio provides little evidence for the suggestion that Damaraland mole-rats, like lions, produce disproportionately male-biased large litters to actively increase the formation of male dispersal coalitions (Packer \& Pusey, 1987). This would suggest that the co-dispersal of closely related Damarland mole-rat males in the wild (Jarvis \& Bennett, 1993; Burland et al., 2004) may comprise individuals from different litters. Increasing the number of male offspring with increasing group size may be sufficient to ensure that more than one male disperser is present when conditions become favourable for breeding dispersal

In conclusion, we observed no helper effects on maternal body mass and litter size in Damaraland mole-rats. While litter size increased with maternal body mass, this did not affect offspring sex ratio. However, in accordance with the LRE hypothesis litters born to pairs were significantly female biased. The opposite was true for groups with more than five female helpers supporting the predictions of the LRC hypothesis, as male offspring are more likely to disperse. In contrast, the number of male pups in a litter increased with the number 
of female but not male helpers. However, the number of helpers of either sex did not affect the number of female offspring. Hence our data suggest that the mechanisms predicted by three hypotheses explaining offspring sex ratio biases (LRE, HR and LRC) can operate simultaneously.

\section{ACKNOWLEDGEMENTS}

We acknowledge funding from the National Research Foundation and the University of Pretoria to NCB. The Department of Nature Conservation Northern Cape and the Department of Environment and Tourism in Namibia are thanked for issuing permits to collect the molerats. The study was approved by the Animal Ethics Committee of the Universities of Cape Town and Pretoria, respectively. The manuscript benefited from the constructive criticism of Kirsty MacLeod and an anonymous reviewer.

\section{References}

Allainé, D. (2000). Sociality, mating system and reproductive skew in marmots: evidence and hypotheses. Behav. Processes 51, 2134.

Allainé, D. (2004). Sex ratio variation in the cooperatively breeding alpine marmot Marmota marmota. Behav. Ecol. 15, 9971002.

Allainé, D., Brondex, F., Graziani, L. \& Coulon, J. (2000). Male-biased sex ratio in litters of Alpine marmots supports the helper repayment hypothesis. Behav. Ecol. 11, 507-514.

Allainé, D. \& Theuriau, F. (2004). Is there an optimal number of helpers in Alpine marmot family groups? Behav. Ecol. 15, 916-924.

Bales, K.L., O'Herron, M., Baker, A.J. \& Dietz, J.M. (2001). Sources of variability in numbers of live births in wild golden lion tamarins (Leontophithecus rosalia). Am. J. Primatol. 54, 211-221.

Begall, S. \& Burda, H. (1998). Reproductive characteristics and growth in the eusocial Zambian Common mole-rat (Cryptomys sp., Bathyergidae). Zeitschrift für Säugetierkd. 63, 297-306.

Bennett, N.C. \& Faulkes, C.G. (2000). African mole-rats: ecology and eusociality.
Cambridge, UK: Cambridge University Press.

Bennett, N.C., Faulkes, C.G. \& Molteno, A.J. (1996). Reproductive suppression in subordinate, non-breeding female Damaraland mole-rats: two components to a lifetime of socially induced infertility. Proc. R. Soc. B 263, 1599-1603.

Bennett, N.C. \& Jarvis, J.U.M. (1988). The social structure and reproductive biology of colonies of the mole-rat Cryptomys damarensis. J. Mammal. 69, 293-302.

Bennett, N.C., Jarvis, J.U.M., Aguilar, G.H. \& McDaid, E.J. (1991). Growth and development in six species of African molerats (Rodentia: Bathyergidae). J. Zool. 225, 13-26.

Bennett, N.C. \& Navarro, R. (1997). Differential growth patterns between successive litters of the eusocial Damaraland mole-rat, Cryptomys damarensis, from Namibia. $J$. Zool. 241, 465-473.

Burland, T.M., Bennett, N.C., Jarvis, J.U.M. \& Faulkes, C.G. (2004). Colony structure and parentage in wild colonies of co-operatively breeding Damaraland mole-rats suggest incest avoidance alone may not maintain reproductive skew. Mol. Ecol. 13, 23712379.

Cameron, E.Z. (2004). Facultative adjustment of mammalian sex ratios in support of the Trivers-Willard hypothesis: evidence for a mechanism. Proc. R. Soc. B 271, 17231728.

Canestrari, D., Vila, M., Marcos, J.M. \& Baglione, V. (2012). Cooperatively breeding carrion crows adjust offspring sex ratio according to group composition. Behav. Ecol. Sociobiol. 66, 1225-1235.

Clark, A.B. (1978). Sex ratio and local resource competition in a prosimian primate. Science (80-. ). 201, 163-165.

Clutton-Brock, T.H. \& Iason, G.R. (1986). Sexratio variation in mammals. Quaterly Rev. Biol. 61, 339-374.

Cooney, R. \& Bennett, N.C. (2000). Inbreeding avoidance and reproductive skew in a cooperative mammal. Proc. R. Soc. B 267, 801-806. 
Dammann, P. \& Burda, H. (2006). Sexual activity and reproduction delay ageing in a mammal. Curr. Biol. 16, R117-R118.

Dammann, P., Šumbera, R., Massmann, C., Scherag, A. \& Burda, H. (2011). Extended longevity of reproductives appears to be common in Fukomys mole-rats (Rodentia, Bathyergidae). PLoS One 6, e18757.

Dušek, A., Bartoš, L. \& Sedláček, F. (2011). Mixed sex allocation strategies in a polytocous mammal, the house mouse (Mus musculus). Behav. Ecol. Sociobiol. 65, 2209-2217.

Emlen, S.T., Emlen, J.M. \& Levin, S.A. (1986). Sex-ratio selection in species with helpersat-the-nest. Am. Nat. 127, 1-8.

Fisher, R.A. (1930). The genetical theory of natural selection. Oxford, UK: Oxford University Press.

Gilchrist, J.S., Otali, E. \& Mwanguhya, F. (2004). Why breed communally? Factors affecting fecundity in a communal breeding mammal: the banded mongoose (Mungos mungo). Behav. Ecol. Sociobiol. 57, 119131.

Gowaty, P.A. \& Lennartz, M.R. (1985). Sex ratios of nestling and fledgling redcockaded woodpeckers (Picoides borealis) favour males. Am. Nat. 126, 347-353.

Griffin, A.S., Sheldon, B.C. \& West, S.A. (2005). Cooperative breeders adjust offspring sex ratios to produce helpful helpers. Am. Nat. 166, 628-632.

Hazell, R.W.A., Bennett, N.C., Jarvis, J.U.M. \& Grif, M. (2000). Adult dispersal in the cooperatively breeding Damaraland mole-rat (Cryptomys damarensis): a case study from the Waterberg region of Namibia. J. Zool. 252, 19-25.

Hickman, G.C. (1979). A live trap and trapping technique for fossorial mammals. South African J. Zool. 14, 9-12.

Isaac, J.L., Krockenberger, A.K. \& Johnson, C.N. (2005). Adaptive sex allocation in relation to life-history in the common brushtail possum, Trichosurus vulpecula. $J$. Anim. Ecol. 74, 552-558.

Jarvis, J.U.M. \& Bennett, N.C. (1993). Eusociality has evolved independently in two genera of bathyergid mole-rats - but occurs in no other subterranean mammal. Behav. Ecol. Sociobiol. 33, 253-260.

Kawalika, M. \& Burda, H. (2006). Giant molerats, Fukomys mechowii, 13 years on the stage. In Subterranean rodents - news from underground: 205-219. Begall, S., Burda, H. \& Schleich, C.E. (Eds). Heidelberg: Springer Berlin Heidelberg.

Koenig, W.D., Pitelka, F.A., Carmen, W.J. \& Mumme, R.L. (1992). The evolution of delayed dispersal in cooperative breeders. Quaterly Rev. Biol. 67, 111-150.

Komdeur, J., Daan, S., Tinbergen, J. \& Mateman, A.C. (1997). Extreme adaptive modification in sex ratio of Seychelles warbler's eggs. Nature 385, 522-525.

Lessells, C. \& Avery, M. (1987). Sex-ratio selection in species with helpers at the nest: some extensions of the repayment model. Am. Nat. 129, 610-620.

Lutermann, H. \& Bennett, N.C. (2008). Strong immune function: a benefit promoting the evolution of sociality? J. Zool. 275, 26-32.

MacLeod, K.J. \& Clutton-Brock, T.H. (2013). No evidence for adaptive sex ratio variation in the cooperatively breeding meerkat, Suricata suricatta. Anim. Behav. 85, 645653.

McNutt, J.W. \& Silk, J.B. (2008). Pup production, sex ratios, and survivorship in African wild dogs, Lycaon pictus. Behav. Ecol. Sociobiol. 62, 1061-1067.

Packer, C. \& Pusey, A.E. (1987). Intrasexual cooperation and the sex ratio in African lions. Am. Nat. 130, 636-642.

Pen, I. \& Weissing, F.J. (2000). Towards a unified theory of cooperative breeding: the role of ecology and life history reexamined. Proc. R. Soc. B 267, 2411-2418.

Rapaport, L.G., Kloc, B., Warneke, M., Mickelberg, J.L. \& Ballou, J.D. (2013). Do mothers prefer helpers? Birth sex-ratio adjustment in captive callitrichines. Anim. Behav. 85, 1295-1302.

Russell, A.F. (2004). Mammals: comparisons and contrasts. In Ecology and evolution of cooperative breeding in birds: 210-227. Koenig, W.D. \& Dickinson, J.L. (Eds). Berkeley, California: Cambrige University Press. 
Russell, A.F., Brotherton, P.N.M., McIlrath, G.M., Sharpe, L.L. \& Clutton-Brock, T.H. (2003). Breeding success in cooperative meerkats: effects of helper number and maternal state. Behav. Ecol. 14, 486-492.

Ryan, C.P., Anderson, W.G., Gardiner, L.E. \& Hare, J.F. (2012). Stress-induced sex ratios in ground squirrels: support for a mechanistic hypothesis. Behav. Ecol. 23, 160-167.

Scantlebury, M., Speakman, J.R., Oosthuizen, M.K., Roper, T.J. \& Bennett, N.C. (2006). Energetics reveals physiologically distinct castes in a eusocial mammal. Nature 440, 795-797.

Schmidt, C.M., Jarvis, J.U.M. \& Bennett, N.C. (2013). The long-lived queen: reproduction and longevity in female eusocial Damaraland mole-rats. African Zool. 48, 193-196.
Sherman, P.W., Lacey, E.A., Reeve, H.K. \& Keller, L. (1995). The eusociality continuum. Behav. Ecol. 6, 102-108.

Sichilima, A.M., Bennett, N.C., Faulkes, C.G. \& Le Comber, S.C. (2008). Evolution of African mole-rat sociality: burrow architecture, rainfall and foraging in colonies of the cooperatively breeding Fukomys mechowii. J. Zool. 275, 276-282.

Silk, J.B. (1984). Local resource competition and the evolution of male-biased sex ratios. $J$. Theor. Biol. 108, 203-213.

Silk, J.B. \& Brown, G.R. (2008). Local resource competition and local resource enhancement shape primate birth sex ratios. Proc. R. Soc. B 275, 1761-1765.

Solomon, N.G. \& French, J.A. (1997). Cooperative Breeding in Mammals. Cambridge: Cambridge University Press.

Trivers, R.L. \& Willard, D.E. (1973). Natural selection of parental ability to vary the sex ratio of offspring. Science 179, 90-93. 


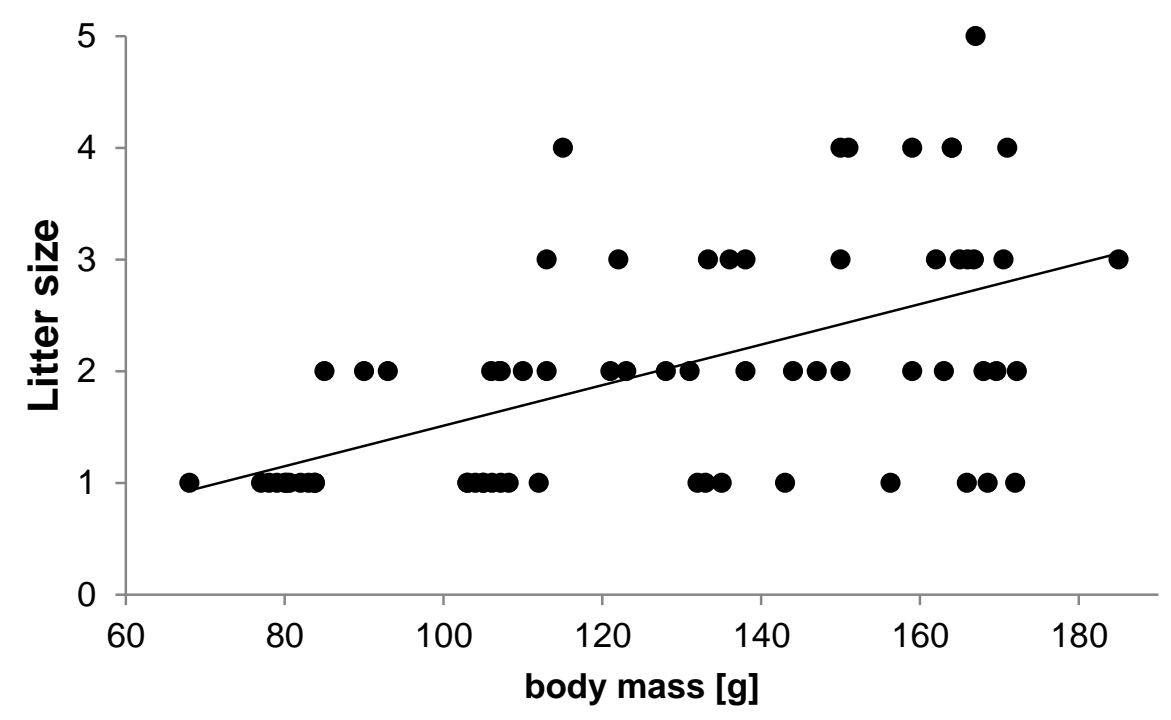

Figure 1. Effects of body mass of the breeding female on litter size 
$\square$ females $\square$ males

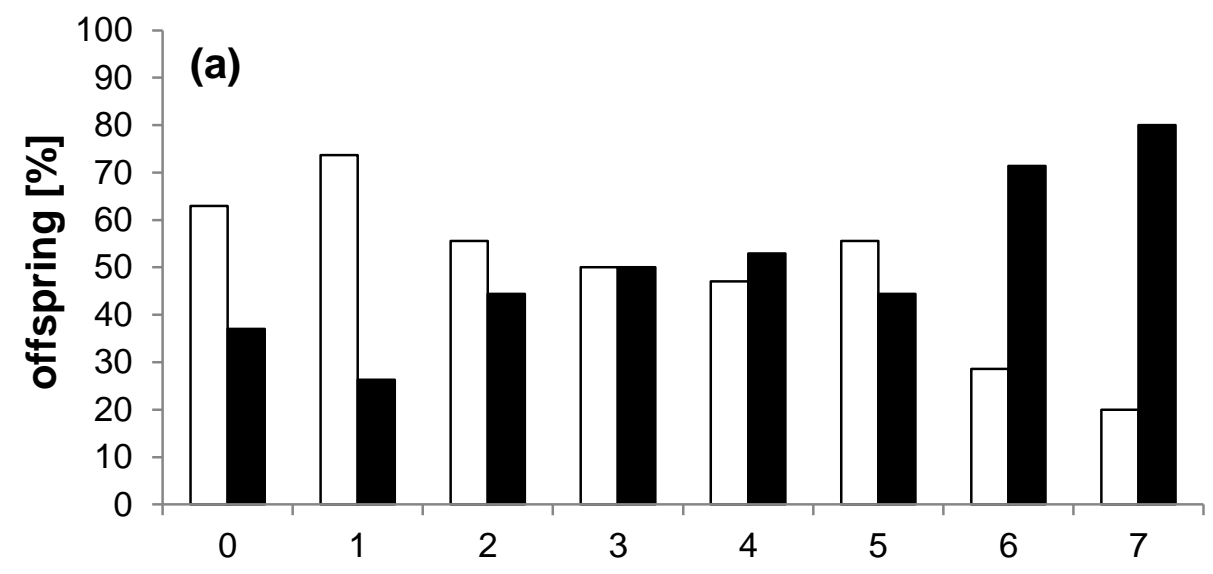

no. of female helpers

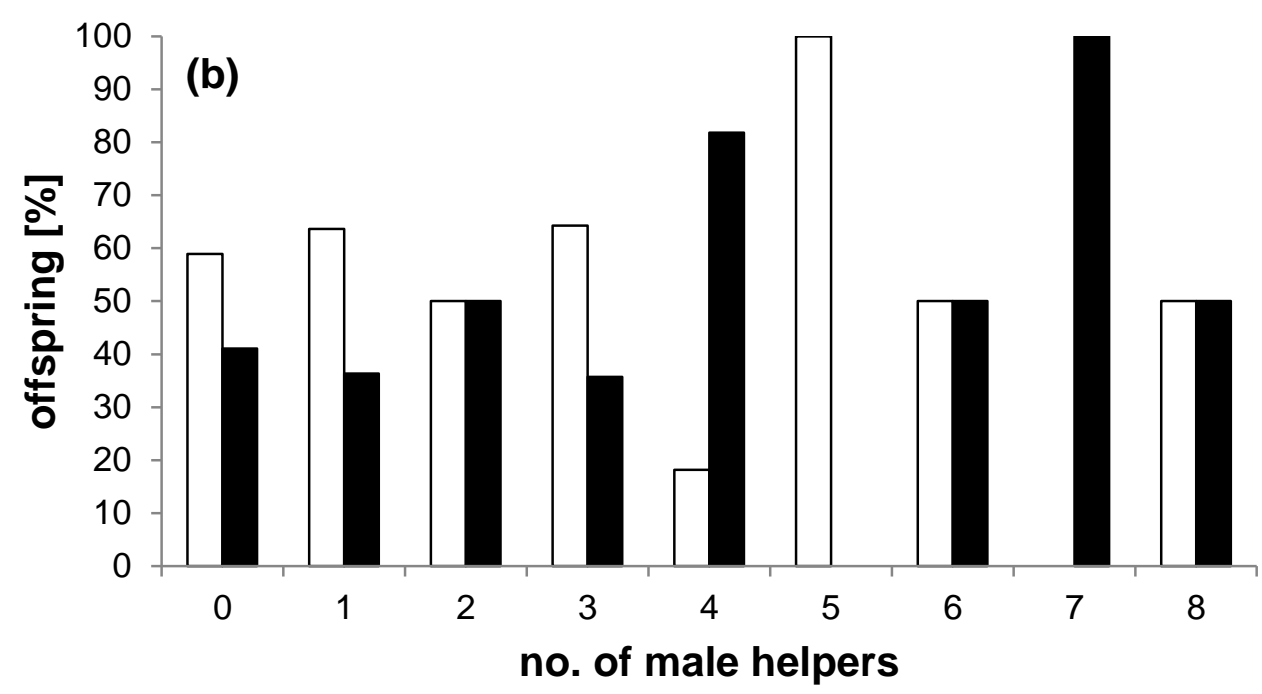

Figure 2. Relationship between the number of a) female and b) male helpers and offspring sex ratio 
Table 1. Predictions and evidence for support of the various hypotheses explaining biased sex ratios among offspring in Damaraland mole-rats

\begin{tabular}{|c|c|c|c|}
\hline \multirow[t]{2}{*}{ Hypothesis } & \multicolumn{2}{|l|}{ Prediction } & \multirow[t]{2}{*}{ Evidence } \\
\hline & $\begin{array}{l}\text { Small groups } \\
\text { (no infrequent helpers*) }\end{array}$ & $\begin{array}{l}\text { Large groups } \\
\text { (infrequent helpers present*) }\end{array}$ & \\
\hline LRE & No sex bias & 우 bias & partially supported \\
\hline HR & No sex bias & No sex bias & partially supported \\
\hline LRC & ฮิ bias & $\widehat{\partial}$ bias & partially supported \\
\hline
\end{tabular}

